\title{
ACYLINDRICAL HYPERBOLICITY OF ARTIN-TITS GROUPS ASSOCIATED WITH TRIANGLE-FREE GRAPHS AND CONES OVER SQUARE-FREE BIPARTITE GRAPHS
}

\author{
MOTOKO KATO ${ }^{\dagger}$ and SHIN-ICHI OGUNI \\ Graduate School of Science and Engineering, Mathematics, Physics and Earth Sciences, Ehime University, \\ 2-5 Bunkyo-cho, Matsuyama, Ehime 790-8577 Japan \\ e-mails: kato.motoko.yy@ehime-u.ac.jp,oguni.shinichi.mb@ehime-u.ac.jp
}

(Received 13 May 2020; revised 16 October 2020; accepted 28 October 2020;

first published online 1 December 2020)

\begin{abstract}
It is conjectured that the central quotient of any irreducible Artin-Tits group is either virtually cyclic or acylindrically hyperbolic. We prove this conjecture for Artin-Tits groups that are known to be CAT(0) groups by a result of Brady and McCammond, that is, Artin-Tits groups associated with graphs having no 3-cycles and Artin-Tits groups of almost large type associated with graphs admitting appropriate directions. In particular, the latter family contains Artin-Tits groups of large type associated with cones over square-free bipartite graphs.
\end{abstract}

2020 Mathematics Subject Classification. Primary 20F65; Secondary 20F36, 20 F67.

1. Introduction. Artin-Tits groups are groups with special finite presentations. Let $\Gamma$ be a finite simple graph with the vertex set $V(\Gamma)$ and the edge set $E(\Gamma)$. An edge $e$ consists of two endvertices, which we denote by $s_{e}$ and $t_{e}$. We suppose that edges $e$ are labeled by integers $m(e)>1$. The Artin-Tits group $A_{\Gamma}$ associated with $\Gamma$ is defined by the following presentation:

$$
A_{\Gamma}=\langle V(\Gamma)| \underbrace{s_{e} t_{e} s_{e} t_{e} \cdots}_{\text {length } m(e)}=\underbrace{t_{e} s_{e} t_{e} s_{e} \cdots}_{\text {length } m(e)} \quad \text { for all } e \in E(\Gamma)\rangle .
$$

Free abelian groups, free groups and braid groups are examples of Artin-Tits groups. If we add relations $v^{2}=1$ to (1.1) for all $v \in V(\Gamma)$, then we get the associated Coxeter group $W_{\Gamma}$. In terms of the properties of $W_{\Gamma}$, we can define important classes of Artin-Tits groups. For example, $A_{\Gamma}$ is said to be of finite type if $W_{\Gamma}$ is finite. Others are said to be of infinite type. We mainly argue on Artin-Tits groups of infinite type.

We can also define classes of Artin-Tits groups in terms of edge labels of $\Gamma . A_{\Gamma}$ is said to be

- right-angled if all edges of $\Gamma$ are labeled by 2 , or

- of large type if all edges of $\Gamma$ are labeled by integers greater than 2 .

\footnotetext{
${ }^{\dagger}$ The first author is supported by JSPS KAKENHI Grant-in-Aid for Research Activity Start-up, Grant Number 19K23406 and JSPS KAKENHI Grant-in-Aid for Young Scientists, Grant Number 20K14311.

$\$$ The second author is supported by JSPS KAKENHI Grant-in-Aid for Young Scientists (B), Grant Number $16 \mathrm{~K} 17595$ and $20 \mathrm{~K} 03590$.
} 
For general Artin-Tits groups, many basic questions are still open (refer to [10]). For example, it is unknown whether the following are equivalent or not for any Artin-Tits group $A_{\Gamma}$ :

(i) $A_{\Gamma}$ is directly indecomposable, that is, it does not decompose as a direct product of two nontrivial subgroups;

(ii) $A_{\Gamma}$ is irreducible, that is, the defining graph $\Gamma$ does not decompose as a join of two non-empty subgraphs such that all edges between them are labeled by 2 .

Note that (i) clearly implies (ii).

Now, we consider questions related to group actions on hyperbolic/non-positively curved spaces. The following is one of the biggest problems on such actions of Artin-Tits groups.

Problem 1.1 ([10, Problem 4]). Which Artin-Tits groups are CAT(0) groups, that is, groups acting geometrically on CAT(0) spaces?

Here, $C A T(0)$ spaces are geodesic spaces where every geodesic triangle is not fatter than the comparison triangle in the Euclidean plane (see [6] for the precise definition). A group action is said to be geometric if the action is proper, cocompact and by isometries.

The following is a related conjecture, which is the main concern of this paper.

CONJECTURE 1.1 ([16, Conjecture B]). The central quotient of every irreducible Artin-Tits group is either virtually cyclic or acylindrically hyperbolic.

The definition of acylindrical hyperbolicity of groups is recalled in Section 2. We can find many applications of acylindrically hyperbolic groups in $[13,21]$ etc. The $n$-strand braid group $B_{n}(n \geq 3)$ is an Artin-Tits group and the central quotient is acylindrically hyperbolic $([3,4,17])$. In addition to this motivating example, Conjecture 1.1 holds for Artin-Tits groups in the following list:

(A1) Artin-Tits groups of finite type ([8]).

(A2) Right-angled Artin-Tits groups $([\mathbf{9}, \mathbf{1 8}])$.

(A3) Two-dimensional Artin-Tits groups such that the associated Coxeter groups are hyperbolic ([19], see also [16] for $A_{\Gamma}$ such that all edges of $\Gamma$ are labeled by integers greater than 4). Note that such a group is characterized as $A_{\Gamma}$ such that every triplet $\left(v_{1}, v_{2}, v_{3}\right)$ of vertices of $\Gamma$ satisfies

$$
\frac{1}{m_{1,2}}+\frac{1}{m_{2,3}}+\frac{1}{m_{3,1}}<1
$$

where

$$
m_{i, j}= \begin{cases}\text { the label of the edge between } v_{i} \text { and } v_{j} & \text { (if } \left.v_{i} \text { and } v_{j} \text { are adjacent }\right) \\ \infty & \text { (if } v_{i} \text { and } v_{j} \text { are not adjacent) }\end{cases}
$$

([20]).

(A4) $A_{\Gamma}$ such that $\Gamma$ is not a join of two non-empty subgraphs ([11], see also [12] for Artin-Tits groups of type FC such that the defining graphs have diameter greater than 2).

To the above list, we add Artin-Tits groups that are known to be CAT(0) groups by a result of Brady and McCammond [5]. We discuss the following two cases. The first case is when $\Gamma$ is triangle-free, that is, $\Gamma$ does not contain 3 -cycles. We do not need any 
restriction on the labels of edges. Such Artin-Tits groups are said to be triangle-free ArtinTits groups.

THEOREM 1.1. For every triangle-free Artin-Tits group $A_{\Gamma}$ such that $\Gamma$ has three or more vertices, the following are equivalent:

(i) $A_{\Gamma}$ is acylindrically hyperbolic.

(ii) $A_{\Gamma}$ is directly indecomposable.

(iii) $A_{\Gamma}$ is irreducible.

(iv) $\Gamma$ is not a complete bipartite graph with all edges labeled by 2.

(v) $\Gamma$ is disconnected, or it contains a 2-path full subgraph with an edge labeled by an integer greater than 2 or a 3-path full subgraph with all edges labeled by 2.

Under either of (i)-(v), and thus all of (i)-(v), $A_{\Gamma}$ is centerless. In particular, Conjecture 1.1 is true for triangle-free Artin-Tits groups.

The second case is the following:

THEOREM 1.2. Let $A_{\Gamma}$ be an Artin-Tits group of almost large type associated with $\Gamma$ with three or more vertices. Suppose that $\Gamma$ can be appropriately directed. Then the following are equivalent:

(i) $A_{\Gamma}$ is acylindrically hyperbolic.

(ii) $A_{\Gamma}$ is directly indecomposable.

(iii) $A_{\Gamma}$ is irreducible.

(iv) $\Gamma$ is not a cone over a graph consisting of only isolated vertices with all edges labeled by 2.

Under either of (i)-(iv), and thus all of (i)-(iv), $A_{\Gamma}$ is centerless. In particular, Conjecture 1.1 is true for Artin-Tits groups of almost large type associated with graphs admitting appropriate directions.

Terminologies "of almost large type" and "appropriately directed" are defined in Section 2. We note that graphs can contain 3-cycles in the setting of Theorem 1.2. As a corollary of Theorem 1.2, we have the following.

COROLLARY 1.3. Let $A_{\Gamma}$ be an Artin-Tits group associated with a cone over a squarefree bipartite graph $\Gamma=\left\{v_{0}\right\} * \Gamma^{\prime}$. Suppose that $\Gamma$ has a 3 -cycle subgraph. Let $\Gamma_{1}^{\prime}$ be the possibly empty subgraph consisting of all isolated vertices in $\Gamma^{\prime}$, and let $\Gamma_{2}^{\prime}=\Gamma^{\prime}-\Gamma_{1}^{\prime}$. Suppose further that every edge in $\Gamma_{2}^{\prime}$ and every edge between $v_{0}$ and $\Gamma_{2}^{\prime}$ are labeled by integers greater than 2. Then $A_{\Gamma}$ is acylindrically hyperbolic, directly indecomposable and centerless. In particular, Conjecture 1.1 is true for such an $A_{\Gamma}$.

Figure 1 shows an example of $\Gamma$ in Corollary 1.3.

By Theorem 1.2, we can also see that Conjecture 1.1 is true for Artin-Tits groups of almost large type associated with square-free graphs (see Corollary 3.4).

Remark 1.1. Theorems 1.1 and 1.2 treated Artin-Tits groups $A_{\Gamma}$ such that $\Gamma$ has three or more vertices, since Conjecture 1.1 is true when $\Gamma$ has less than three vertices. In fact, if $\Gamma$ has less than three vertices, it satisfies one of the following: (1) $\Gamma$ has only one vertex, (2) $\Gamma$ has exactly two vertices and no edges, and (3) $\Gamma$ has exactly two vertices and an edge. In the first case, $A_{\Gamma} \cong \mathbb{Z}$, and thus the central quotient is trivial. In the second case, $A_{\Gamma} \cong \mathbb{F}_{2}$, which is hyperbolic. Since its center is trivial, the central quotient of $A_{\Gamma}$ is acylindrically hyperbolic. In the third case, when the edge label is $2, A_{\Gamma} \cong \mathbb{Z}^{2}$, which is reducible. When 


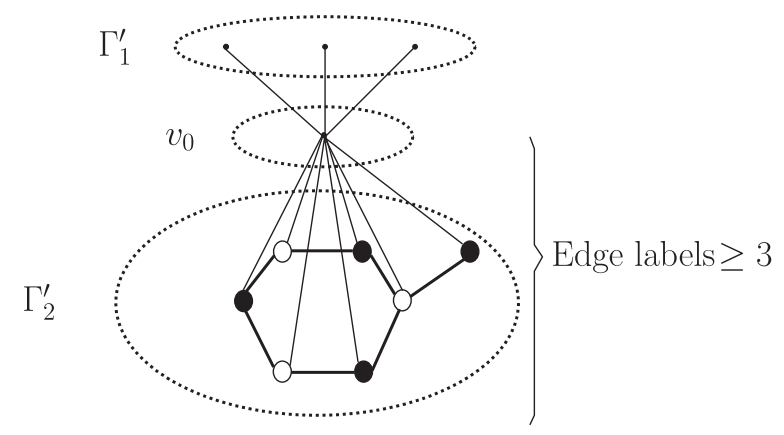

Figure 1. The join of graphs $v_{0}$ and $\Gamma_{1}^{\prime} \cup \Gamma_{2}^{\prime}$.

2

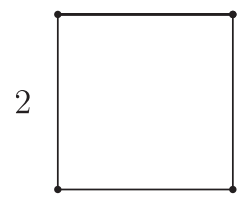

3
3

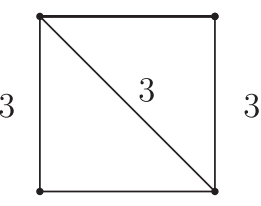

3

Figure 2. Two examples of $\Gamma$ such that $A_{\Gamma}$ is not in (A1)-(A4).

the edge label is greater than $2, A_{\Gamma}$ has an infinite cyclic center and its central quotient is $\mathbb{Z} / m \mathbb{Z} * \mathbb{Z} / 2 \mathbb{Z}$ for odd $m$ and $\mathbb{Z} / \frac{m}{2} \mathbb{Z} * \mathbb{Z}$ for even $m$ (see [16]). These free products are virtually $\mathbb{F}_{2}$ and thus hyperbolic.

We compare Artin-Tits groups in Theorem 1.1 and Corollary 1.3 with (A1)-(A4). Our Artin-Tits groups are not necessarily of finite type, right-angled or with the associated Coxeter groups being hyperbolic. A triangle-free Artin-Tits group is possibly associated with a join. Also, a cone over a graph is a join. Figure 2 shows a triangle-free graph and a cone over a square-free bipartite graph such that associated Artin-Tits groups are not in (A1)-(A4).

Finally, we consider one of the basic questions on algebraic properties of Artin-Tits groups. When an irreducible Artin-Tits group is of finite type, the center is known to be infinite cyclic $([7,14])$. For irreducible Artin-Tits groups of infinite type, it is conjectured that the center is trivial $([\mathbf{1 0}, \mathbf{1 5}])$. When $\Gamma$ is not a cone, it is known that $A_{\Gamma}$ is centerless ([11]). Theorem 1.1 and Theorem 1.2 give affirmative partial answers to this conjecture. In particular, Corollary 1.3 claims that some Artin-Tits groups associated with cones are centerless.

We give an outline of this paper. Section 2 contains preliminaries on acylindrically hyperbolic groups, Artin-Tits groups, and Brady-McCammond's CAT(0) spaces. Section 3 contains proofs of Theorems 1.1 and 1.2. Our strategy is to answer the following problem: if an Artin-Tits group acts geometrically on a CAT(0) space, does it have a rank-one isometry? Such a strategy is based on relations between rank-one isometries on CAT(0) spaces and acylindrical hyperbolicity of groups $([2,22])$ and was used in previous works on ArtinTits groups (for example, [16]). In the proofs of the main theorems, we observe geometric actions of Artin-Tits groups on CAT(0) spaces, constructed by Brady and McCammond [5]. We detect group elements acting as rank-one isometries on the CAT( 0$)$ spaces. 


\section{Preliminaries.}

2.1. Acylindrically hyperbolic groups. Hereafter, we always assume that group actions on metric spaces are by isometries. We recall the definition of acylindrically hyperbolic groups.

Definition $2.1([4,21])$. An isometric action of a group $G$ on a metric space $(X, d)$ is acylindrical if for every $\varepsilon \geq 0$, there exist $R \geq 0$ and $N \geq 0$ such that every $x, y \in X$ with $d(x, y) \geq R$ satisfy

$$
|\{g \in G \mid d(x, g x) \leq \varepsilon, d(y, g y) \leq \varepsilon\}| \leq N .
$$

A group $G$ is acylindrically hyperbolic if $G$ acts acylindrically and non-elementarily on a (Gromov-) hyperbolic space.

Examples and basic properties of acylindrically hyperbolic groups can be found in [21].

Definition 2.2 (cf. [6]). An isometry $\gamma$ on a metric space $(X, d)$ is hyperbolic if there exists a point $x \in X$ satisfying $d(x, \gamma x)=\inf _{x^{\prime} \in X} d\left(x^{\prime}, \gamma x^{\prime}\right)>0$. When $X$ is a CAT(0) space, $\gamma$ is hyperbolic if and only if it acts by a translation on a geodesic line $l_{\gamma}$ in $X$. We call $l_{\gamma}$ an axis of $\gamma$ ([6, Theorem II-6.8]). $\gamma$ is rank one if it is hyperbolic and its axis does not bound a flat half-plane.

THEOREM 2.1 ([22, Theorem 1.3]). If a group $G$ acts properly on a proper CAT(0) space with a rank-one isometry, then $G$ is either virtually cyclic or acylindrically hyperbolic.

2.2. Artin-Tits groups and Brady-McCammond's CAT(0) spaces. Let $\Gamma$ be a finite simple graph with edges labeled by integers greater than 1 . The associated ArtinTits group $A_{\Gamma}$ is defined by the standard presentation (1.1). A graph is said to be directed if every edge $e$ is identified with an ordered pair $\left(s_{e}, t_{e}\right)$ of endvertices. When $\Gamma$ is directed, $A_{\Gamma}$ admits another presentation.

Lemma 2.2 ([5, Section 5, Definition $\left.\left.\left(G_{\Gamma}\right)\right]\right)$. Let $\Gamma$ be a finite simple directed graph with the vertex set $V(\Gamma)$ and the edge set $E(\Gamma)$. Suppose that edges e are labeled by integers $m(e)>1$. Then $A_{\Gamma}$ admits a presentation with the generating set:

$$
V(\Gamma) \cup\left\{x_{e}\right\}_{e \in E(\Gamma)} \cup\left\{\alpha_{e, 3}, \ldots, \alpha_{e, m(e)}\right\}_{e \in E(\Gamma), m(e) \geq 3}
$$

and relations

$$
x_{e}=s_{e} t_{e}, x_{e}=t_{e} s_{e}
$$

for every $e \in E(\Gamma)$ with $m(e)=2$, and

$$
x_{e}=s_{e} t_{e}, x_{e}=t_{e} \alpha_{e, 3}, \ldots, x_{e}=\alpha_{e, i} \alpha_{e, i+1}, \ldots, x_{e}=\alpha_{e, m(e)} s_{e}
$$

for every $e \in E(\Gamma)$ with $m(e) \geq 3$.

Let $K_{\Gamma}$ be the presentation complex associated with the presentation of $A_{\Gamma}$ in Lemma 2.2. $K_{\Gamma}$ has a unique vertex $o$, a directed 1-cell for each generator and a 2-cell for each relation in (2.3) and (2.4). We denote by $\operatorname{Pr}: \widetilde{K}_{\Gamma} \rightarrow K_{\Gamma}$ the projection from the universal cover $\widetilde{K}_{\Gamma}$ onto $K_{\Gamma}$. The 1-skeleton of $\widetilde{K}_{\Gamma}$ can be identified with the Cayley graph of $A_{\Gamma}$ on the generators (2.2). We fix such an identification and let $\widetilde{o} \in \widetilde{K}_{\Gamma}$ be the vertex corresponding to the identity element of $A_{\Gamma}$. Figure 3 shows 2-cells in the universal cover 

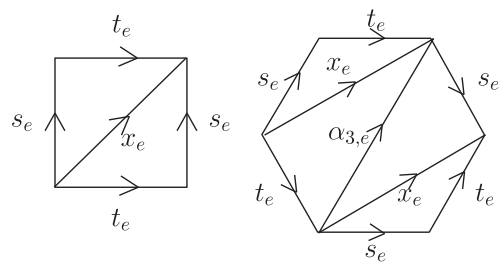

Figure 3. 2-cells in $\widetilde{K}_{\Gamma}$ corresponding to edges of $\Gamma$ labeled by 2 and 3.
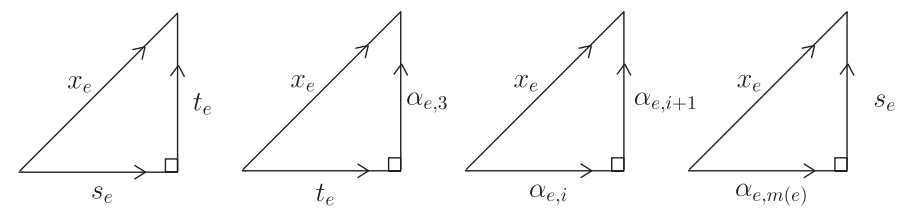

Figure 4. 2-cells of $\widetilde{K}_{\Gamma}$ isometric to an Euclidean isosceles right triangle.
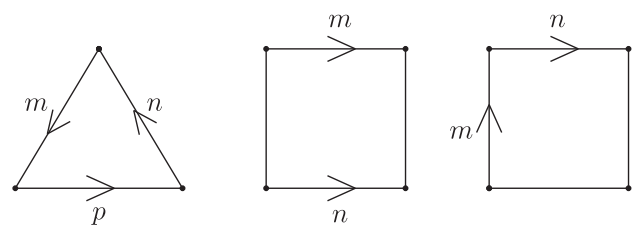

Figure 5. Directed 3-cycles and 4-cycles. Labels $m, n$, and $p$ are greater than 2. Unlabeled undirected edges can admit any label greater than 1 and any direction.

$\widetilde{K}_{\Gamma}$ of $K_{\Gamma}$. In [5], Brady and McCammond showed that $\widetilde{K}_{\Gamma}$ can be given a metric to be an $A_{\Gamma}$-equivariant $\mathrm{CAT}(0)$ space under some combinatorial assumptions on $\Gamma$.

Let us consider two families of Artin-Tits groups. The first one is the family of triangle-free Artin-Tits groups.

THEOREM 2.3 ([5, Theorem 6]). Let $A_{\Gamma}$ be a triangle-free Artin-Tits group. Let us assign $\Gamma$ an arbitrary direction. Then $\widetilde{K}_{\Gamma}$ has a metric satisfying the following:

- all 2-cells are isometric to a Euclidean isosceles right triangle, and

- all 1-cells corresponding to generators in $\left\{x_{e}\right\}_{e \in E(\Gamma)}$ are the longest and of length $3 \sqrt{2}$.

Moreover, $\widetilde{K}_{\Gamma}$ with this metric is a proper $C A T(0)$ space. The action of $A_{\Gamma}$ on $\widetilde{K}_{\Gamma}$ is geometric.

Figure 4 shows the identification of 2-cells with Euclidean triangles in Theorem 2.3.

The second one is the family of Artin-Tits groups of almost large type associated with graphs admitting appropriate directions. Here, an Artin-Tits group $A_{\Gamma}$ is said to be of almost large type if the defining graph $\Gamma$ satisfies the following two conditions:

(1) For every 3-cycle in $\Gamma$, all edges are labeled by integers greater than 2 .

(2) For every 4-cycle in $\Gamma$, at least two edges are labeled by integers greater than 2 .

In addition, we say that such a $\Gamma$ admits an appropriate direction or can be appropriately directed if $\Gamma$ can be directed such that each 3- (resp. 4-) cycle is directed in the same way as one of 3- (resp. 4-) cycles in Figure 5. We note that $\Gamma$ can admit 3-cycles.

THEOREM 2.4 ([5, Theorem 7 and Remark on p. 9]). Let $A_{\Gamma}$ be an Artin-Tits group of almost large type and $\Gamma$ admits an appropriate direction. Let us assign $\Gamma$ an appropriate 


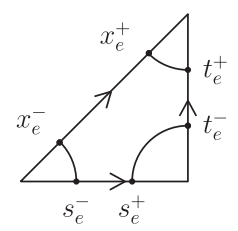

Figure 6. Intersection points of 1-cells $x_{e}, s_{e}$, and $t_{e}$ with $L_{\Gamma}$.

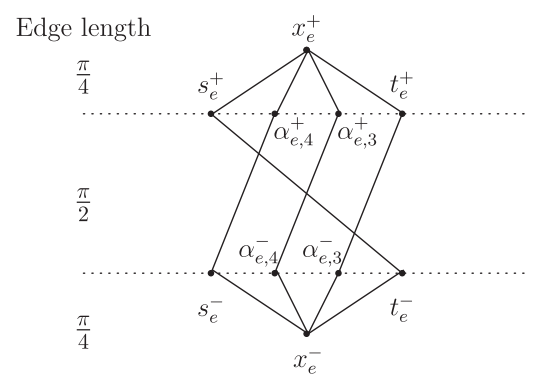

Figure 7. A part of $L_{\Gamma}$ related to an edge $e$ labeled by 4 in Theorem 2.3.

direction. Then $\widetilde{K}_{\Gamma}$ has a metric such that all 2-cells are isometric to a Euclidean equilateral triangle with side length 3. Moreover, $\widetilde{K}_{\Gamma}$ with this metric is a proper $C A T(0)$ space. The action of $A_{\Gamma}$ on $\widetilde{K}_{\Gamma}$ is geometric.

In Theorems 2.3 and 2.4, we assign $K_{\Gamma}$ a metric $d_{K_{\Gamma}}$ such that it is locally isometric to $\widetilde{K}_{\Gamma}$. We often observe the link $L_{\Gamma}=\left\{z \in K_{\Gamma} \mid d_{K_{\Gamma}}(o, z)=1\right\}$ of the unique vertex $o$ in $K_{\Gamma}$. Note that $L_{\Gamma}$ is regarded as a graph. Indeed, each 1-cell of $K_{\Gamma}$ corresponds to two vertices of $L_{\Gamma}$, and each corner of a 2-cell of $K_{\Gamma}$ corresponds to an edge of $L_{\Gamma}$. We assign $L_{\Gamma}$ the path metric induced by the metric of $K_{\Gamma}$. Then, the distance between adjacent vertices of $L_{\Gamma}$ is the angle between corresponding 1-cells at $o$ in $K_{\Gamma}$.

We consider the setting of Theorem 2.3. For every directed 1-cell $c$ of $K_{\Gamma}$, two intersection points with $L_{\Gamma}$ are named $c^{-}$and $c^{+}$in order, see Figure 6. We draw $L_{\Gamma}$ following [5], see Figure 7. In $L_{\Gamma}$, every edge connected to a vertex $x_{e}^{+}$or $x_{e}^{-}$(a "top" or "bottom" edge in Figure 7) is of length $\pi / 4$. The other edges ("middle" edges in Figure 7) are of length $\pi / 2$. By noting that $\Gamma$ is triangle-free, we can confirm that $L_{\Gamma}$ does not contain nontrivial loops of length less than $2 \pi$. This fact is a key ingredient of the proof of Theorem 2.3 ([5, Theorem 6]).

Under the setting of Theorem 2.4, we can discuss everything in a similar way. We note that all the edges of $L_{\Gamma}$ are of length $\pi / 3$.

\section{Main results.}

3.1. Triangle-free Artin-Tits groups. In this section, we prove Theorem 1.1.

Lemma 3.1. Let $A_{\Gamma}$ be a triangle-free Artin-Tits group and let $\Gamma$ be assigned an arbitrary direction, as in Theorem 2.3. Suppose further that $\Gamma$ contains one of the following directed graphs as a full subgraph:

(1) the 2-path directed graph $\Gamma_{m, n}$ as in Figure 8, and

(2) the 3-path directed graph $\Gamma_{2,2,2}$ as in Figure 8.

Then $A_{\Gamma}$ is acylindrically hyperbolic. 

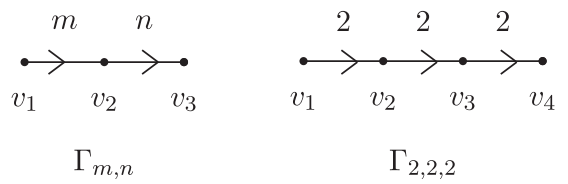

Figure 8. A 2-path directed graph $\Gamma_{m, n}$ with edges labeled by $m \geq 2$ and $n \geq 3$, and a 3-path directed graph $\Gamma_{2,2,2}$ with edges labeled by 2 .
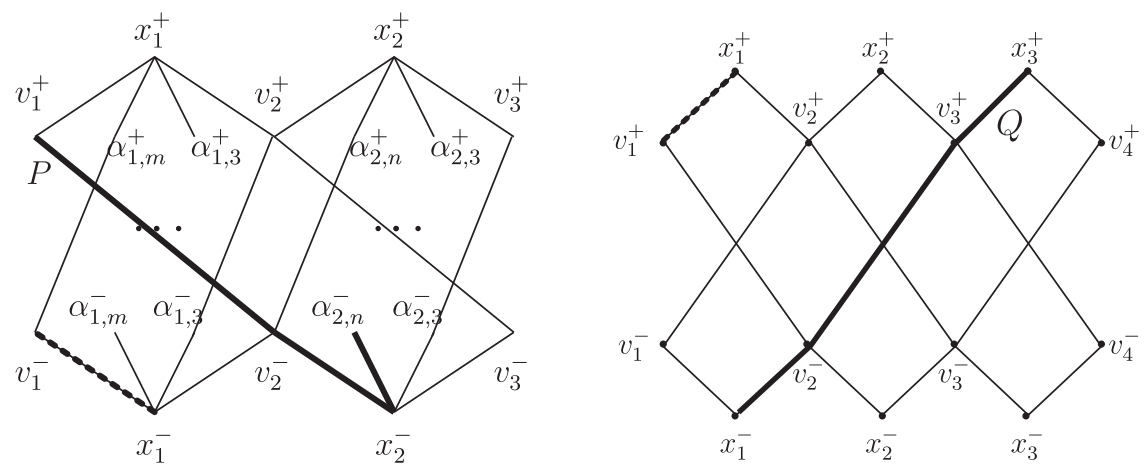

Figure 9. $L_{\Gamma_{m, n}}$ and $L_{\Gamma_{2,2,2}}$.

Proof. Let $\widetilde{K}_{\Gamma}$ be the $A_{\Gamma}$-equivariant CAT(0) space in Theorem 2.3. Let $L_{\Gamma}$ be the link of the unique vertex $o$ of $K_{\Gamma}$. We find a rank-one isometry in $A_{\Gamma}$.

(1) We discuss the case where $\Gamma$ contains $\Gamma_{m, n}$ as a full subgraph. By Lemma 2.2, $A_{\Gamma_{m, n}}$ has the following presentation:

$$
\left|\begin{array}{l}
v_{1}, v_{2}, v_{3}, x_{1}, x_{2}, \\
\alpha_{1,3}, \ldots, \alpha_{1, m}, \\
\alpha_{2,3}, \ldots, \alpha_{2, n}
\end{array}\right| \begin{aligned}
& x_{1}=v_{1} v_{2}, x_{1}=v_{2} \alpha_{1,3}, \ldots, x_{1}=\alpha_{1, m} v_{1}, \\
& x_{2}=v_{2} v_{3}, x_{2}=v_{3} \alpha_{2,3}, \ldots, x_{2}=\alpha_{2, n} v_{2}
\end{aligned} \mid
$$

Let $K_{\Gamma_{m, n}} \subset K_{\Gamma}$ be the presentation complex of (3.1). Let $L_{\Gamma_{m, n}} \subset L_{\Gamma}$ be the link in $K_{\Gamma_{m, n}}$. We draw $L_{\Gamma_{m, n}}$ on the left-hand side of Figure 9.

We show that $\alpha_{2, n} v_{3} v_{1}$ acts as a rank-one isometry on $\widetilde{K}_{\Gamma}$. First, we find an axis of $\alpha_{2, n} v_{3} v_{1}$. In $K_{\Gamma}$, let $l$ be the concatenation of 1-cells $\alpha_{2, n}, v_{3}$, and $v_{1}$ in this order. We note that 1 -cells of $K_{\Gamma}$ are loops based at $o$. We show that $l$ is a local geodesic. Since $l$ is geodesic around any point of $l$ except $o$, we investigate $l$ around $o$ and show that:

$$
d_{L_{\Gamma}}\left(v_{1}^{+}, \alpha_{2, n}^{-}\right), d_{L_{\Gamma}}\left(\alpha_{2, n}^{+}, v_{3}^{-}\right), d_{L_{\Gamma}}\left(v_{3}^{+}, v_{1}^{-}\right) \geq \pi .
$$

To see $d_{L_{\Gamma}}\left(v_{1}^{+}, \alpha_{2, n}^{-}\right) \geq \pi$, we find a shortest path $P$ from $v_{1}^{+}$to $\alpha_{2, n}^{-}$uniquely in $L_{\Gamma_{m, n}}$, which is of length $\pi$ (see the bold line in Figure 9). This path $P$ is the unique shortest one even in $L_{\Gamma}$. Indeed, if we have a shortest path $P^{\prime}$ from $v_{1}^{+}$to $\alpha_{2, n}^{-}$in $L_{\Gamma}$ through $L_{\Gamma}-L_{\Gamma_{m, n}}$, then $P^{\prime}$ should go out from $L_{\Gamma_{m, n}}$ at a point in $\left\{v_{i}^{ \pm}\right\}_{i=1,2,3}$ and come back into $L_{\Gamma_{m, n}}$ at another point in $\left\{v_{i}^{ \pm}\right\}_{i=1,2,3}$. Since $\Gamma$ is simple and $\Gamma_{m, n}$ is a full subgraph, every path through $\Gamma-\Gamma_{m, n}$ in $\Gamma$ between different vertices in $\left\{v_{i}\right\}_{i=1,2,3}$ contains at least two edges. It follows that every path through $L_{\Gamma}-L_{\Gamma_{m, n}}$ between different points of $\left\{v_{i}^{ \pm}\right\}_{i=1,2,3}$ is of length greater than or equal to $\pi$, see Figure 10. This shows that the length of $P^{\prime}$ is greater than $\pi$. In particular 
$\Gamma^{\prime}$
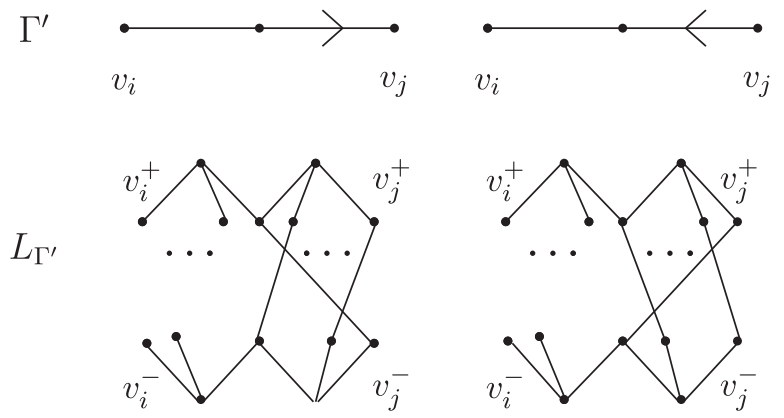

Figure 10. Assuming that $v_{i}$ and $v_{j}$ are not adjacent in $\Gamma$, the distance between different points of $\left\{v_{i}^{ \pm}, v_{j}^{ \pm}\right\}$in $L_{\Gamma}$ is at least $\pi$. The minimum $\pi$ occurs only in cases where $v_{i}$ and $v_{j}$ are connected by a 2-path $\Gamma^{\prime}$ in $\Gamma$.

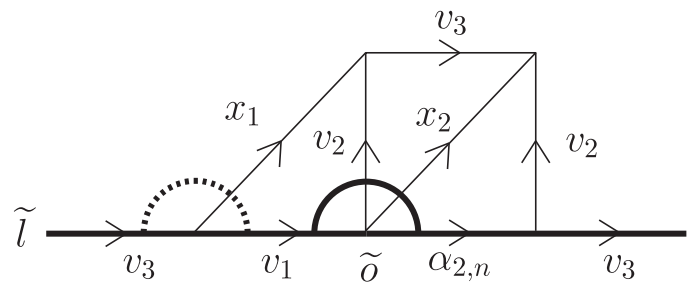

Figure 11. An axis $\tilde{l}$ of $\alpha_{2, n} v_{3} v_{1}$.

we have

$$
d_{L_{\Gamma}}\left(v_{1}^{+}, \alpha_{2, n}^{-}\right)=d_{L_{\Gamma m, n}}\left(v_{1}^{+}, \alpha_{2, n}^{-}\right)=\pi .
$$

Similarly, we confirm that:

$$
d_{L_{\Gamma}}\left(\alpha_{2, n}^{+}, v_{3}^{-}\right)=d_{L_{\Gamma m, n}}\left(\alpha_{2, n}^{+}, v_{3}^{-}\right)=\pi .
$$

Also, according to Figure $9, d_{L_{\Gamma_{m, n}}}\left(v_{3}^{+}, v_{1}^{-}\right)>\pi$. Therefore,

$$
d_{L_{\Gamma}}\left(v_{3}^{+}, v_{1}^{-}\right) \geq \pi .
$$

Here, the minimum $\pi$ occurs only when $v_{1}$ and $v_{3}$ are connected by a 2-path $\Gamma^{\prime}$ in Figure 10. By (3.3), (3.4), and (3.5), we have (3.2). It follows that $l$ is geodesic around $o$. Indeed, assume that a geodesic from $v_{1}^{+}$to $\alpha_{2, n}^{-}$, a geodesic from $\alpha_{2, n}^{+}$to $v_{3}^{-}$or a geodesic from $v_{3}^{+}$ to $v_{1}^{-}$does not pass through $o$. Then a triplet $\left(v_{1}^{+}, o, \alpha_{2, n}^{-}\right),\left(\alpha_{2, n}^{+}, o, v_{3}^{-}\right)$, or $\left(v_{3}^{+}, o, v_{1}^{-}\right)$contributes to a non-collapsing geodesic triangle as vertices. Since $K_{\Gamma}$ is piecewise Euclidean and $\operatorname{CAT}(0)$ around $o$, three interior angles of such a triangle must be less than $\pi$, contrary to (3.2) (cf. discussions in the proof of Theorem 15 of [1]). Therefore, $l$ is locally geodesic. Hence, the lift $\widetilde{l}$ through $\widetilde{o}$ is an axis of $\alpha_{2, n} v_{3} v_{1}$, see Figure 11.

Next, we show that $\widetilde{l}$ does not bound a flat half-plane. On the contrary, we assume that $\widetilde{l}$ bounds a flat half-plane $E$. By $\operatorname{Pr}: \widetilde{K}_{\Gamma} \rightarrow K_{\Gamma}$, the unit semicircle $C \subset E$ centered at $\widetilde{o}$ is isometric to the path $P$ of length $\pi$ from $v_{1}^{+}$to $\alpha_{2, n}^{-}$in $L_{\Gamma}$ (see the bold semicircle in Figure 11). It follows that $C$ goes through lifts of $v_{1}^{+}, v_{2}^{-}, x_{2}^{-}$, and $\alpha_{2, n}^{-}$. Therefore, $E$ contains 2-cells corresponding to relations $x_{1}=v_{1} v_{2}, x_{2}=v_{2} v_{3}$, and $x_{2}=\alpha_{2, n} v_{2}$ around $\widetilde{o}$. The unit semicircle in $E$ centered at $v_{1}^{-1}(\widetilde{o})$ should go through lifts of $v_{1}^{-}, x_{1}^{-}$and $v_{3}^{+}$(see the dotted semicircle in Figure 11). This is impossible, since there is no path of length $\pi$ in $L_{\Gamma}$ from $v_{1}^{-}$to $v_{3}^{+}$through $x_{1}^{-}$(see Figure 9). 


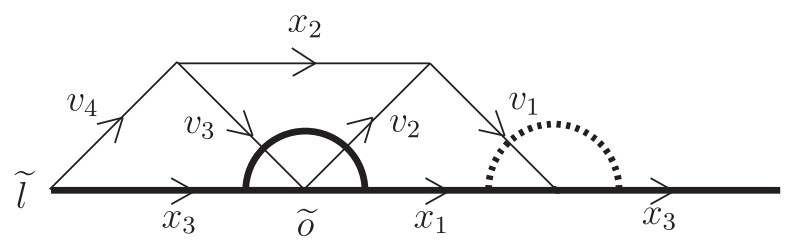

Figure 12. An axis $\tilde{l}$ of $x_{1} x_{3}$.

Since we detected a rank-one isometry $\alpha_{2, n} v_{3} v_{1}$ in $A_{\Gamma}$, Theorem 2.1 shows that $A_{\Gamma}$ is acylindrically hyperbolic.

(2) We discuss the case where $\Gamma$ contains $\Gamma_{2,2,2}$ as a full subgraph. The argument is similar to (1). According to Lemma 2.2, $A_{\Gamma_{2,2,2}}$ has the following presentation:

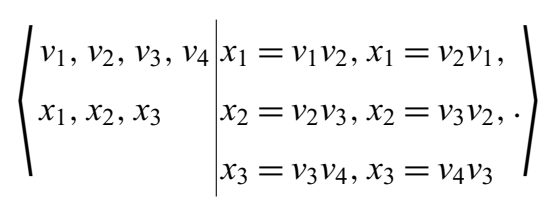

Let $K_{\Gamma_{2,2,2}} \subset K_{\Gamma}$ be the presentation complex of (3.6). Let $L_{\Gamma_{2,2,2}} \subset L_{\Gamma}$ be the link in $K_{\Gamma_{2,2,2}}$. We draw $L_{\Gamma_{2,2,2}}$ on the right-hand side of Figure 9 .

We show that $x_{1} x_{3}$ acts as a rank-one isometry on $\widetilde{K}_{\Gamma}$. Let $l$ be the concatenation of 1-cells $x_{1}$ and $x_{3}$ in $K_{\Gamma}$. As in (1), we can find a shortest path $Q$ from $x_{3}^{+}$to $x_{1}^{-}$ uniquely in $L_{\Gamma}$ (see the bold line in Figure 9). This path $Q$ is of length $\pi$. In particular, we have $d_{L_{\Gamma}}\left(x_{3}^{+}, x_{1}^{-}\right)=\pi$. Similarly, we confirm that $d_{L_{\Gamma}}\left(x_{1}^{+}, x_{3}^{-}\right)=\pi$. Therefore, $l$ is $\underset{\sim}{\mathrm{q}}$ local geodesic, and the lift $\widetilde{l}$ through $\widetilde{o}$ is an axis of $x_{1} x_{3}$, see Figure 12. Now assume that $\tilde{l}$ bounds a flat half-plane $E$. By $\operatorname{Pr}: \widetilde{K}_{\Gamma} \rightarrow K_{\Gamma}$, the unit semicircle $C \subset E$ centered at $\widetilde{o}$ is isometric to the path $Q$ of length $\pi$ from $x_{3}^{+}$to $x_{1}^{-}$in $L_{\Gamma}$ (see the bold semicircle in Figure 12). It follows that $C$ goes through lifts of $x_{3}^{+}, v_{3}^{+}, v_{2}^{-}$, and $x_{1}^{-}$. Therefore, $E$ contains lifts of 2-cells corresponding to relations $x_{3}=v_{4} v_{3}, x_{2}=v_{3} v_{2}$, and $x_{1}=v_{2} v_{1}$ around $\widetilde{o}$. Then, the unit semicircle in $E$ centered at $x_{1}(\widetilde{o})$ should go through lifts of $x_{1}^{+}, v_{1}^{+}$, and $x_{3}^{-}$(see the dotted semicircle in Figure 12). This is impossible, since there is no path of length $\pi$ in $L_{\Gamma}$ from $x_{1}^{+}$to $x_{3}^{-}$through $v_{1}^{+}$(see Figure 9). Theorem 2.1 shows that $A_{\Gamma}$ is acylindrically hyperbolic.

LEMMA 3.2. If $\Gamma$ is a connected triangle-free graph with more than one vertex, then either $\Gamma$ is a complete bipartite graph or contains the 3-path graph as a full subgraph.

Proof. Let $\Gamma$ be a connected triangle-free graph with more than one vertex. If $\Gamma$ contains an $n$-cycle of $n \geq 5$ as a full subgraph, then $\Gamma$ contains a 3-path subgraph of the $n$-cycle as a full subgraph. Otherwise, $\Gamma$ does not have odd cycles and thus is a bipartite graph. We divide the vertex set of $\Gamma$ into two non-empty subsets $V_{W}$ and $V_{B}$ such that every edge connects a vertex in $V_{W}$ and one in $V_{B}$. If $\Gamma$ is not a complete bipartite graph, then there exist vertices $v_{W} \in V_{W}$ and $v_{B} \in V_{B}$ of graph distance greater than 2, and every shortest path from $v_{W}$ to $v_{B}$ is a full subgraph. For such a path, any 3-subpath is a full subgraph of $\Gamma$. An example of $\Gamma$ is shown in Figure 13, where the division of the vertex set is drawn as white/black coloring of vertices.

Proof of Theorem 1.1. Let $\Gamma$ be a finite simple graph with edges labeled by integers greater than 1. Suppose that $\Gamma$ is triangle-free with at least three vertices. 


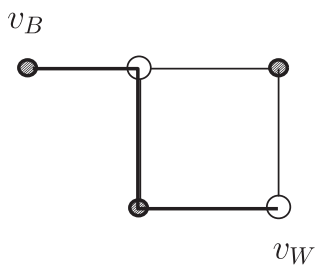

Figure 13. A bipartite graph $\Gamma$ which is not complete.

[(i) $\Rightarrow$ (ii)] Suppose that $A_{\Gamma}$ is acylindrically hyperbolic. In general, an acylindrically hyperbolic group does not decompose as a direct product of two infinite groups ([21]). Since $K_{\Gamma}$ in Theorem 2.3 is a finite $K\left(A_{\Gamma}, 1\right)$ space, $A_{\Gamma}$ is torsion-free. It follows that $A_{\Gamma}$ does not decompose as a direct product of two nontrivial subgroups.

[(ii) $\Rightarrow$ (iii)] We prove the contrapositive. If $\Gamma$ is a join of two non-empty subgraphs $\Gamma_{1}$ and $\Gamma_{2}$ such that all edges between them are labeled by 2 , then $A_{\Gamma}$ is the direct product of $A_{\Gamma_{1}}$ and $A_{\Gamma_{2}}$.

[(iii) $\Rightarrow$ (iv)] We prove the contrapositive. If $\Gamma$ is a complete bipartite graph with all edges labeled by 2 , then $\Gamma$ is a join of two non-empty subgraphs without any edge $\Gamma_{1}$ and $\Gamma_{2}$ such that all edges between them are labeled by 2 . Therefore, $A_{\Gamma}$ is reducible.

$[(i v) \Rightarrow(v)]$ Suppose that $\Gamma$ is not a complete bipartite graph with all edges labeled by 2. Under this assumption, it is enough to consider the following three cases: (1) $\Gamma$ is not connected, (2) $\Gamma$ is connected and at least one edge of $\Gamma$ is labeled by an integer greater than 2 , and (3) $\Gamma$ is connected and all edges of $\Gamma$ are labeled by 2 . In the second case, $\Gamma$ contains a 2-path full subgraph with an edge labeled by an integer greater than 2 . In the third case, we can apply Lemma 3.2, since $\Gamma$ is a connected triangle-free graph with more than one vertex, It follows that $\Gamma$ either is a complete bipartite graph or contains a 3-path full subgraph. By the assumption, $\Gamma$ contains a 3-path full subgraph with all edges labeled by 2 .

$\left[(\mathrm{v}) \Rightarrow\right.$ (i)] First, if $\Gamma$ is disconnected, then $A_{\Gamma}$ decomposes as a free product of two infinite subgroups, and thus acylindrically hyperbolic. Second, suppose that $\Gamma$ contains a 2-path full subgraph with an edge labeled by an integer greater than 2 . Let us assign a direction to the subgraph in the same way as $\Gamma_{m, n}$ in Figure 8. We direct other edges arbitrarily. With this direction, $\Gamma$ satisfies the assumptions in Lemma 3.1. Lemma 3.1 shows that $A_{\Gamma}$ is acylindrically hyperbolic. Finally, suppose that $\Gamma$ contains a 3-path full subgraph with all edges labeled by 2 . Let us assign a direction to the subgraph in the same way as $\Gamma_{2,2,2}$ in Figure 8. Other edges are directed arbitrarily. With this direction, $\Gamma$ satisfies the assumptions in Lemma 3.1. Lemma 3.1 shows that $A_{\Gamma}$ is acylindrically hyperbolic.

Suppose that $A_{\Gamma}$ is acylindrically hyperbolic. Since an acylindrically hyperbolic group does not admit an infinite center ([21]), the center of $A_{\Gamma}$ is finite. Since $A_{\Gamma}$ has a finite $K\left(A_{\Gamma}, 1\right)$ space $K_{\Gamma}, A_{\Gamma}$ is torsion-free. It follows that the center of $A_{\Gamma}$ is trivial.

Let $A_{\Gamma}$ be an irreducible triangle-free Artin-Tits group. When $\Gamma$ has three or more vertices, the central quotient of $A_{\Gamma}$ is $A_{\Gamma}$ itself, which is acylindrically hyperbolic. When $\Gamma$ has less than three vertices, see Remark 1.1.

3.2. Artin-Tits groups associated with cones over square-free bipartite graphs. In this section, we prove Theorem 1.2 and Corollary 1.3. 


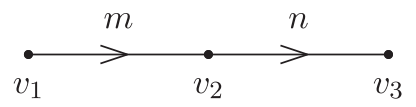

Figure 14. A 2-path directed graph $\Gamma^{\prime}$ with edges labeled by $m, n \geq 3$.

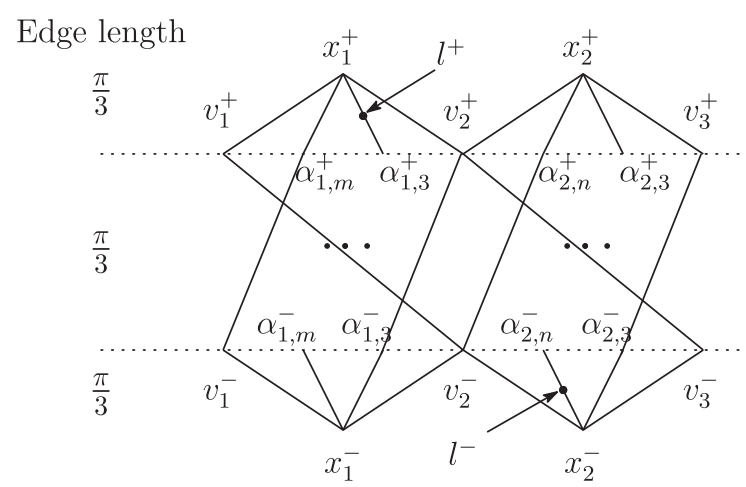

Figure 15. The subgraph $L_{\Gamma^{\prime}}$ of $L_{\Gamma}$ corresponding to the 2-path subgraph $\Gamma^{\prime}$ of $\Gamma$, and two points $l^{ \pm}$.

Lemma 3.3. Let $A_{\Gamma}$ be an Artin-Tits group of almost large type. Suppose that $\Gamma$ is a complete bipartite graph with all edges labeled by 2 . Then $\Gamma$ is a cone over a graph consisting of only isolated vertices with all edges labeled by 2.

Proof. Assume that $\Gamma$ is not a cone. Then, $\Gamma$ has a square with all edges labeled by 2 , which contradicts the assumption that $A_{\Gamma}$ is of almost large type.

Proof of Theorem 1.2. [(i) $\Rightarrow$ (ii) $\Rightarrow$ (iii) $\Rightarrow$ (iv)] We repeat the same argument as in the proof of Theorem 1.1 .

[(iv) $\Rightarrow$ (i)] Let $A_{\Gamma}$ be triangle-free. By Lemma 3.3, (iv) in Theorem 1.2 implies (iv) in Theorem 1.1. Therefore, according to Theorem $1.1, A_{\Gamma}$ is acylindrically hyperbolic.

We consider the case where $\Gamma$ has a 3-cycle. Let us assign $\Gamma$ an appropriate direction. Let $\widetilde{K}_{\Gamma}$ be the $A_{\Gamma}$-equivariant $\mathrm{CAT}(0)$ space in Theorem 2.4 . Let $L_{\Gamma}$ be the link of the unique vertex $o$ of $K_{\Gamma}$. We find a rank-one isometry in $A_{\Gamma}$. We fix a 3-cycle of $\Gamma$. Then, as a subgraph of the 3-cycle, we take a 2-path subgraph $\Gamma^{\prime}$ in Figure 14. By Lemma 2.2, $A_{\Gamma^{\prime}}$ has the following presentation:

$$
\left|\begin{array}{l}
v_{1}, v_{2}, v_{3}, x_{1}, x_{2}, \\
\alpha_{1,3}, \ldots, \alpha_{1, m}, \\
\alpha_{2,3}, \ldots, \alpha_{2, n}
\end{array}\right| \begin{aligned}
& x_{1}=v_{1} v_{2}, x_{1}=v_{2} \alpha_{1,3}, \ldots, x_{1}=\alpha_{1, m} v_{1}, \\
& x_{2}=v_{2} v_{3}, x_{2}=v_{3} \alpha_{2,3}, \ldots, x_{2}=\alpha_{2, n} v_{2}
\end{aligned} \mid
$$

Since $\Gamma^{\prime}$ is not a full subgraph of $\Gamma, A_{\Gamma^{\prime}}$ is not a subgroup of $A_{\Gamma}$. On the other hand, the presentation complex $K_{\Gamma^{\prime}}$ of (3.7) is a subcomplex of $K_{\Gamma}$. Let $L_{\Gamma^{\prime}} \subset L_{\Gamma}$ be the link in $K_{\Gamma^{\prime}}$, see Figure 15. As shown in Figure 16, we take a directed loop $l$ in $K_{\Gamma^{\prime}}$, which intersects with $L_{\Gamma^{\prime}}$ at two points $l^{+}$and $l^{-}$. In $L_{\Gamma^{\prime}}$, the distance between $l^{+}$and $l^{-}$is greater than $\pi$, see Figure 15. Even in $L_{\Gamma}$, the distance between $l^{+}$and $l^{-}$is greater than $\pi$. Indeed, a shortest path from $l^{+}$to $l^{-}$in $L_{\Gamma}$ through $L_{\Gamma}-L_{\Gamma^{\prime}}$ should go out from $L_{\Gamma^{\prime}}$ at a point in $\left\{v_{i}^{ \pm}\right\}_{i=1,2,3}$, passes at least one edge in $L_{\Gamma}-L_{\Gamma^{\prime}}$, and comes back into $L_{\Gamma^{\prime}}$ at another point 


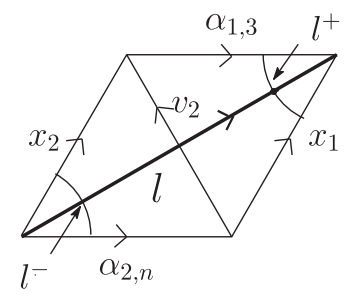

Figure 16. A directed loop $l$ in $K_{\Gamma^{\prime}}$ and its intersection points with $L_{\Gamma^{\prime}}$.

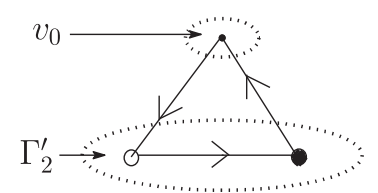

Figure 17. A directed 3-cycle in $\Gamma$.

in $\left\{v_{i}^{ \pm}\right\}_{i=1,2,3}$. The length of such a path is greater than $\pi$, since the distance between $l^{ \pm}$ and $\left\{v_{i}^{ \pm}\right\}_{i=1,2,3}$ in $L_{\Gamma^{\prime}}$ is greater than $\pi / 3$. Therefore,

$$
d_{L_{\Gamma}}\left(l^{+}, l^{-}\right)>\pi
$$

As in the proof of Lemma 3.1, $l$ is a local geodesic around $o$, and the lift $\tilde{l}$ through $\widetilde{o}$ is an axis of $\gamma=x_{2} \alpha_{1,3}$ in $A_{\Gamma}$ (see Figure 16). Assume that $\widetilde{l}$ bounds a flat half-plane $E$. By $\operatorname{Pr}: \widetilde{K}_{\Gamma} \rightarrow K_{\Gamma}$, the unit semicircle in $E$ centered at $\widetilde{o}$ on $\widetilde{l}$ is isometric to a path of length $\pi$ from $l^{+}$to $l^{-}$in $L_{\Gamma}$, contrary to (3.8).

When $A_{\Gamma}$ is acylindrically hyperbolic, the same discussion as in the proof of Theorem 1.1 shows that the center of $A_{\Gamma}$ is trivial. Similarly, Conjecture 1.1 can be confirmed.

Proof of Corollary 1.3. Let $\Gamma$ satisfy assumptions in Corollary 1.3. By assumptions on edge labels of $\Gamma, A_{\Gamma}$ is of almost large type. We show that $\Gamma$ can be appropriately directed. Note that $\Gamma_{2}^{\prime}$ is bipartite. We color vertices of $\Gamma_{2}^{\prime}$ by black and white such that every edge in $\Gamma_{2}^{\prime}$ connects a white vertex and a black vertex. We give a direction to every edge $e$ of $\Gamma$ as follows. If $e$ is in $\left\{v_{0}\right\} * \Gamma_{1}^{\prime}, e$ is directed arbitrarily. If $e$ is in $\Gamma_{2}^{\prime}, e$ goes from a white vertex to a black vertex. Otherwise, $e$ goes from $v_{0}$ to a white vertex or goes from a black vertex to $v_{0}$. Then, every 3-cycle is the one in Figure 5, as shown in Figure 17. Note that $\Gamma_{2}^{\prime}$ has no 4-cycles. Since every 4-cycle shares two edges with such a directed 3 -cycle, it is the same as the rightmost 4-cycle in Figure 5. Hence, $\Gamma$ is appropriately directed. Since $\Gamma$ satisfies the condition (iv) in Theorem 1.2, $A_{\Gamma}$ satisfies (i), (ii), and (iii) in Theorem 1.2.

Let us state one more corollary of Theorem 1.2.

COROLlaRY 3.4. Let $A_{\Gamma}$ be an Artin-Tits group of almost large type associated with $\Gamma$ with three or more vertices. Suppose that $\Gamma$ is square-free, that is, $\Gamma$ does not contain 4-cycles. When $A_{\Gamma}$ is irreducible, it is acylindrically hyperbolic, directly indecomposable, and centerless. In particular, Conjecture 1.1 is true for such an $A_{\Gamma}$.

Proof. If $\Gamma$ is triangle-free, then we use Theorem 1.1. Otherwise, every two triangles of $\Gamma$ either share only a vertex or are disjoint, since $\Gamma$ is square-free. We can assign a 
direction to all triangles as in Figure 5. When other edges of $\Gamma$ are directed arbitrarily, $\Gamma$ is appropriately directed. We apply Theorem 1.2.

ACKNOWLEDGEMENTS. The authors would like to thank the anonymous reviewers for comments on the previous version of this paper.

\section{REFERENCES}

1. W. Ballmann, Singular spaces of nonpositive curvature, in Sur les groupes hyperboliques d'après Mikhael Gromov (Bern, 1988), Progress in Mathematics, vol. 83 (Birkhäuser Boston, Boston, MA, 1990), 189-201.

2. M. Bestvina, K. Bromberg and K. Fujiwara, Constructing group actions on quasi-trees and applications to mapping class groups, Publ. Math. Inst. Hautes Études Sci. 122 (2015), 1-64.

3. M. Bestvina and K. Fujiwara, Bounded cohomology of subgroups of mapping class groups, Geom. Topol. 6 (2002), 69-89. $281-300$

4. B. H. Bowditch, Tight geodesics in the curve complex, Invent. Math. 171(2) (2008),

5. T. Brady and J. P. McCammond, Three-generator Artin groups of large type are biautomatic, J. Pure Appl. Algebra 151(1) (2000), 1-9.

6. M. R. Bridson and A. Haefliger, Metric spaces of non-positive curvature, Grundlehren der Mathematischen Wissenschaften [Fundamental Principles of Mathematical Sciences], vol. 319 (Springer-Verlag, Berlin, 1999). $245-271$

7. E. Brieskorn and K. Saito, Artin-Gruppen und Coxeter-Gruppen, Invent. Math. 17 (1972),

8. M. Calvez and B. Wiest, Acylindrical hyperbolicity and Artin-Tits groups of spherical type, Geom. Dedicata 191 (2017), 199-215.

9. P.-E. Caprace and M. Sageev, Rank rigidity for CAT(0) cube complexes, Geom. Funct. Anal. 21(4) (2011), 851-891.

10. R. Charney, Problems related to Artin groups (2008). http://people.brandeis.edu/ $\sim$ charney/papers/Artin_probs.pdf.

11. R. Charney and R. Morris-Wright, Artin groups of infinite type: trivial centers and acylindrical hyperbolicity, Proc. Amer. Math. Soc. 147(9) (2019), 3675-3689.

12. I. Chatterji and A. Martin, A note on the acylindrical hyperbolicity of groups acting on CAT( 0$)$ cube complexes, in Beyond hyperbolicity (Hagen M., Webb R. and Wilton H., Editors), London Mathematical Society Lecture Note Series, vol. 454 (Cambridge University Press, Cambridge, 2019), 160-178.

13. F. Dahmani, V. Guirardel and D. Osin, Hyperbolically embedded subgroups and rotating families in groups acting on hyperbolic spaces, Mem. Amer. Math. Soc. 245(1156) (2017), v+152.

14. P. Deligne, Les immeubles des groupes de tresses généralisés, Invent. Math. 17 (1972), 273-302.

15. E. Godelle and L. Paris, Basic questions on Artin-Tits groups, in Configuration spaces, CRM Series, vol. 14 (Ed. Norm., Pisa, 2012), 299-311.

16. T. Haettel, XXL type artin groups are CAT(0) and acylindrically hyperbolic, preprint, 2019, arXiv: 1905.11032.

17. U. Hamenstädt, Bounded cohomology and isometry groups of hyperbolic spaces, J. Eur. Math. Soc. (JEMS) 10(2) (2008), 315-349.

18. S.-H. Kim and T. Koberda, The geometry of the curve graph of a right-angled Artin group, Int. J. Algebra Comput. 24(2) (2014), 121-169.

19. A. Martin and P. Przytycki, Acylindrical actions for two-dimensional Artin groups of hyperbolic type, preprint, 2019, arXiv: 1906.03154.

20. G. Moussong, Hyperbolic Coxeter groups. Thesis $(\mathrm{PhD})-$ The Ohio State University (ProQuest LLC, Ann Arbor, MI, 1988).

22. A. Sisto, Contracting elements and random walks, J. Reine Angew. Math. 742 (2018), $79-114$. 